\title{
Development of Eugenol-Embedded Calcium Citrate Nanoparticles as a Local Anesthetic Agent
}

\author{
Sarocha Cherdchom, Wittawat Keawsongsaeng, Wanida Buasorn, Natchanon Rimsueb, \\ Prompong Pienpinijtham, Amornpun Sereemaspun, Rojrit Rojanathanes, * and Pornanong Aramwit*
}

Cite This: ACS Omega 2021, 6, 28880-28889

Read Online

ABSTRACT: Eugenol is a major phenolic component derived from clove oil with potential medical applications. Of particular interest, it has been used as a therapeutic agent in topical applications because of its analgesic and local anesthetic properties. However, topical formulations of eugenol produce skin irritation, which limits its clinical applications. One promising strategy to overcome this disadvantage is by using a biocompatible material that could be an appropriate topical vehicle for eugenol. Researchers have recently focused on the development of eugenol-embedded calcium citrate nanoparticles (Eu-CaCit NPs) without adverse effects. The Eu-CaCit NPs were developed as a topical delivery system and their biocompatibility and penetration ability were evaluated. Eu-CaCit NPs at $1.2 \mathrm{mg} / \mathrm{mL}$ did not show cytotoxicity effects in human cells. Moreover, the Eu-CaCit NPs presented the ability to penetrate the dermis layer of the human intact skin following $12 \mathrm{~h}$ exposure. All the results concluded that Eu-CaCit NPs have shown a potential as a carrier for topical delivery of eugenol. These novel nanoparticles represent a promising alternative for topical application of local anesthetic with natural pain relievers.

\section{INTRODUCTION}

Pain is a common complication in clinical practice. Patients suffering from pain exhibit dysfunctional behaviors and a poor quality of life. ${ }^{1,2}$ Local anesthetics have been used in clinical practices to reduce the painful site and for producing a local pain-relieving effect in patients. The effective assessment and management of pain and suffering can improve not only biological but also psychological and social variables. ${ }^{3}$ Many local anesthetic agents including lidocaine, bupivacaine, and ropivacaine are some of the most commonly used agents. ${ }^{4}$ These drugs are used in operative procedures, acute pain, chronic pain, and other uses. However, adverse reactions are often associated with the use of these agents. ${ }^{5,6}$ Therefore, there is a growing demand for preventing and managing undesired pain without adverse effects using natural compounds.

Eugenol (4-allyl-2-methoxyphenol) is a naturally occurring phenol essential oil extracted from cloves of the Syzygium genus. It is a versatile natural compound that has many potential properties such as antioxidant, anti-inflammation, neuroprotective effects, and antimicrobial activities. ${ }^{7-9}$ Moreover, eugenol is known to be useful as a local anesthetic in dentistry because of its ability to allay tooth pain through inhibition of the voltage-gated sodium channel (VGSC) and activation of the transient receptor potential vanilloid subtype 1 (TRPV1). ${ }^{10}$ Nevertheless, the traditional use of eugenol as a local anesthetic in a painful site such as the intact skin has been limited because of its unwanted side effects. Topical treatment of eugenol is followed by localized irritation of the skin and is significantly limited by its poor water solubility. ${ }^{11,12}$ Owing to its potential anesthetic properties, the drug delivery system has been designed to overcome the physiological barriers of the skin and improve the therapeutic efficacy of eugenol via topical medication.

Topical drug delivery systems appear to be an interesting alternative to provide great potential for non- or minimally invasive drug delivery. ${ }^{13}$ Moreover, the large surface area of skin has the advantage of having interesting sites for topical medication. ${ }^{14}$ The stratum corneum is the main barrier for drugs to pass through the skin. Several approaches have been made to either disrupt or weaken the stratum corneum to improve skin delivery. Accomplishing high and constant drug penetration through the skin has now become possible with the development of a topical drug delivery system using nanoparticles (NPs) as drug carriers.

NPs can be used a promising carrier for topical drug delivery systems due to their ability of drug skin penetration and skin targeting. ${ }^{14,15}$ Moreover, NPs have the realistic potential to improve the stability of hydrophobic drugs and reduce toxicity

Received: July 19, 2021

Accepted: October 14, 2021

Published: October 25, 2021 
by using biocompatible nanomaterials. ${ }^{16-18}$ The emergence of NP technologies has also made it possible to develop a new drug carrier in topical therapy. We have been studying ways to facilitate the penetration of a wide range of drugs through the stratum corneum using new biocompatible materials. In a recent study, our research group had successfully synthesized a novel type of NP based on calcium citrate NPs (CaCit NPs), which presented a high stability and biocompatibility for drug delivery applications. ${ }^{19}$ Following the advancement, the main objective of this work is to investigate the potential use of $\mathrm{CaCit}$ NPs as a vehicle for the topical application of eugenol. Novel eugenol-embedded CaCit NPs (Eu-CaCit NPs) were prepared as well as the penetration ability of Eu-CaCit NPs into the human skin was determined. The Eu-CaCit NPs present high potential as a therapeutic agent for medicinal applications without adverse effects.

\section{MATERIALS AND METHODS}

2.1. Chemicals and Materials. Eugenol was purchased from Sigma-Aldrich. Calcium chloride dihydrate $(\geq 98.0 \%)$, trisodium citrate dihydrate $(\geq 99.0 \%)$, and sodium hydroxide $(\mathrm{NaOH}, \geq 97.0 \%)$ were all obtained from Merck (Germany). Fluorescein 5(6)-isothiocyanate isomer I (FITC, $\geq 90.0 \%$ ) was purchased from TCI Chemicals (Japan).

2.2. Synthesis of Eu-CaCit NPs. Eugenol (100 mg) was dissolved in $0.375 \mathrm{M} \mathrm{NaOH}$ solution $(2.00 \mathrm{~mL})$ and treated with $1.00 \mathrm{M}$ trisodium citrate solution $(5.00 \mathrm{~mL})$ in one portion. The resulting solution was stirred at room temperature for $10 \mathrm{~min}$ then $2.00 \mathrm{M}$ calcium chloride solution (3.25 $\mathrm{mL}$ ) was added while stirring. A milky suspension was formed. This suspension was stirred at room temperature for an additional $30 \mathrm{~min}$. The mixture was centrifuged and the remaining solid was washed with deionized water (DI water) for three times and then dried in vacuum overnight, yielding a white solid as the desired product.

2.3. Preparation of FITC-Eu-CaCit NPs. In a $15 \mathrm{~mL}$ centrifuge tube, a solution of FITC $(1.0 \mathrm{mg})$ in $5.0 \mathrm{mM} \mathrm{NaOH}$ solution $(5 \mathrm{~mL})$ was slowly added into the colloid of Eu-CaCit $\mathrm{NPs}$ in DI water $(100 \mathrm{mg}$ in $5 \mathrm{~mL}$ ) while stirring. After completion of the addition, the mixture was continuously stirred for an additional $1 \mathrm{~h}$ and then washed by centrifugation for $10 \mathrm{~min}$. The remaining solid was washed with DI water three times and then dried in vacuum overnight, yielding a yellowish-white solid as the desired product.

2.4. Scanning Electron Microscopy (SEM). The characterization of NPs was carried out using SEM operated at $20 \mathrm{kV}$ (JSM-6480LV).

2.5. Thermogravimetric Analysis (TGA). The percentages of eugenol in CaCit NPs were evaluated by TGA with a STA 409 PC TA system from 25 to $800{ }^{\circ} \mathrm{C}$. The sample was placed in a platinum pan and heated at a constant rate of $20 \mathrm{~K} /$ min in a constant flow of nitrogen.

2.6. X-ray Powder Diffraction (XRD). XRD patterns were measured using a Rigaku SmartLab $30 \mathrm{kV}$ diffractometer equipped with a fixed monochromator and a $\mathrm{Cu} \mathrm{K} \alpha$ radiation source, which was set at an accelerating voltage of $40 \mathrm{kV}$ and an applied current of $30 \mathrm{~mA}$.

2.7. Fourier Transform Infrared Spectroscopy (FTIR). All FTIR spectra were collected by a Thermo Scientific Nicolet iS5 Fourier transformed infrared spectrometer in an attenuated total reflection (ATR) mode using an iD7 ATR accessory, which were recorded by a deuterated triglycine sulfate (DTGS) detector with a scan number of 16 and a spectral resolution of $4 \mathrm{~cm}^{-1}$. All spectra were presented without any modification, except normalization.

2.8. Zeta Potential Measurement. Zeta potential of the outer surface of each layer was measured using Zetasizer Nano ZS (Malvern, Southborough, MA, USA) in order to ensure the alternate deposition of $\mathrm{CaCit}$ NPs. Each value so obtained was in effect the average of three parallel measurements.

2.9. Cell Culture. Human keratinocytes (HaCaT) and human primary fibroblasts were cultured and maintained to confluence in a growth medium of DMEM supplemented with $10 \%$ fetal bovine serum and $1 \%$ antibiotic (Gibco, USA). Cultures were maintained in an incubator at $37{ }^{\circ} \mathrm{C}$ in a humidified atmosphere of $5 \% \mathrm{CO}_{2}$. Human primary fibroblasts were obtained as a kind gift from Dr. Jiraroch Meevassana, Center of Excellence in Burn and Wound Care, Chulalongkorn University, Bangkok, Thailand.

2.10. Cytotoxicity Measurements. Cytotoxicity was assessed with a PrestoBlue reagent assay (Gibco, USA). Human keratinocytes and fibroblasts were seeded onto 96-well plates at a density of $1 \times 10^{4}$ or $5 \times 10^{3}$ cells/well, respectively, and allowed to reach confluence in $24 \mathrm{~h}$. Cells were treated with eugenol $(50 \mu \mathrm{g} / \mathrm{mL})$ and NPs $(1.2 \mathrm{mg} / \mathrm{mL})$ for 24,48 , and $72 \mathrm{~h}$, and a PrestoBlue reagent solution was then added to each well. After incubation, the fluorescence intensity was measured at an excitation of $560 \mathrm{~nm}$ and an emission of 590 $\mathrm{nm}$ by using a microplate reader (Thermo Fisher, Varioskan Flash, England). The cell viability was expressed as a percentage relative to the untreated cells with treated groups. The cell morphology was observed under an inverted microscope (Nikon, Japan).

2.11. Cellular Uptake Study. Human keratinocytes and fibroblasts were cultured in $35 \mathrm{~mm}$ tissue culture dishes and incubated under $5 \%(\mathrm{v} / \mathrm{v})$ relative humidity at $37{ }^{\circ} \mathrm{C}$ for $24 \mathrm{~h}$. The FITC-Eu-CaCit NPs were added and incubated as above for $24 \mathrm{~h}$. The uptake of the samples was also observed by a confocal laser scanning microscope (Zeiss LSM 800, Carl Zeiss, Oberkochen, Germany) at an excitation wavelength of $488 \mathrm{~nm}$ and optical modes.

2.12. Drug Release Study. To study the release mechanism of the drug delivery system, $40 \mathrm{mg}$ of Eu-CaCit NPs was suspended in $2.00 \mathrm{~mL}$ of $1 \times$ phosphate buffered saline (PBS) added to ethanol (EtOH; PBS/EtOH = 2:1) with different $\mathrm{pH}$ levels $(\mathrm{pH} 4.8,5.5$, and 7.4). The vials were incubated in a water bath at $37^{\circ} \mathrm{C}$. Subsequently, $1.00 \mathrm{~mL}$ of the sample was withdrawn at predetermined intervals of time. The supernatant $(1.00 \mathrm{~mL})$ was collected at each release time point. Eugenol released into PBS was quantified by measuring the absorbance at $282 \mathrm{~nm}$ using a microplate reader (Thermo Fisher, Varioskan Flash, England). The concentrations of eugenol were determined from calibration curves.

2.13. Skin Penetration Study. The methodology of this study was approved by the Ethics Committee of the hospital and Faculty of Medicine, Chulalongkorn University (IRB374/ 62). All skin samples were used with permission from the Director of King Chulalongkorn Memorial Hospital. The soft embalmed cadaver skin was obtained from the Chula Soft Cadaver Surgical Training Center, Faculty of Medicine, Chulalongkorn University. The skin penetration was conducted using Franz diffusion cells. A piece of human skin was shaved, cut into $20 \mathrm{~cm}^{2}$ round pieces, and placed in water at 37 ${ }^{\circ} \mathrm{C}$ for $1 \mathrm{~min}$ to wash away the oil on the skin surface. The human skin sample was then placed in the middle of the Franz diffusion cell and fixed by clamping. The receptor fluid was 
a

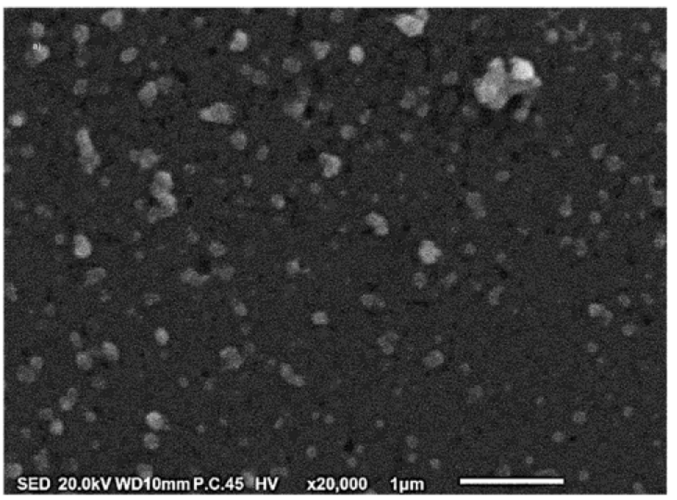

b

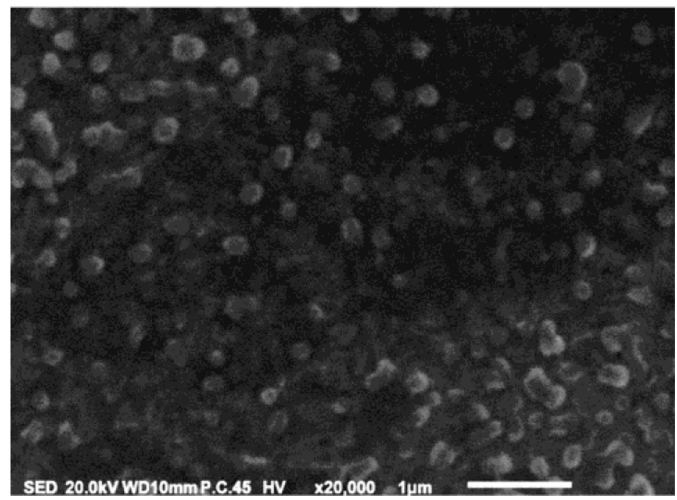

Figure 1. SEM images of Eu-CaCit NPs (a) and FITC-Eu-CaCit NPs (b) at 20,000 magnifications.

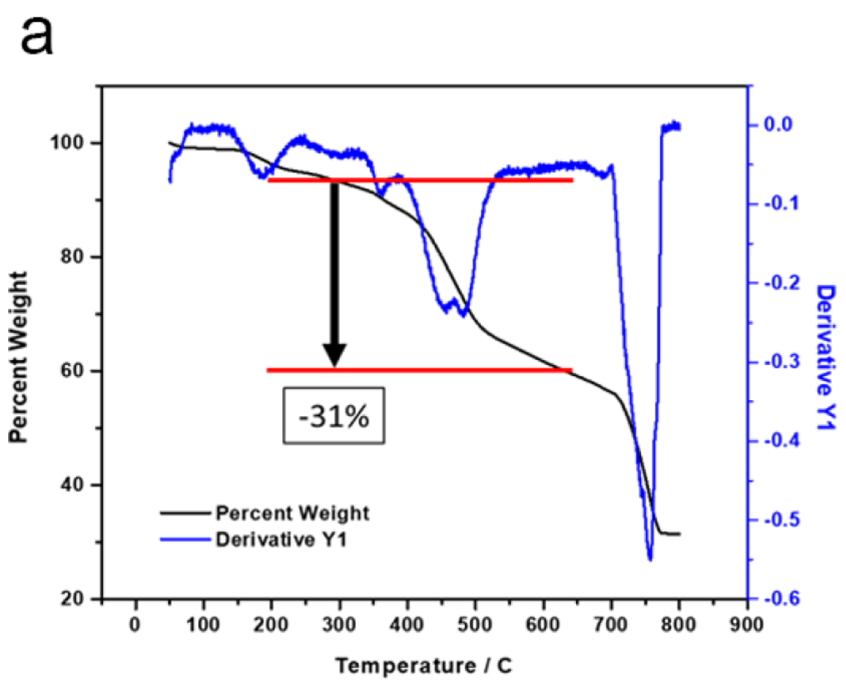

b

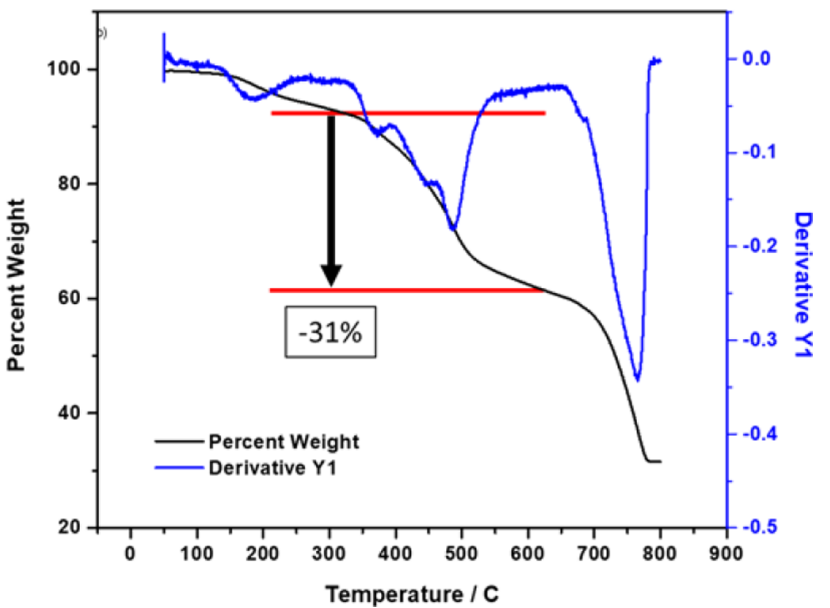

Figure 2. Thermogravimetric analysis and derivative curves of Eu-CaCit NPs (a) and FITC-Eu-CaCit NPs (b).

composed of $1 \times$ PBS added to ethanol $(\mathrm{EtOH} ; \mathrm{PBS} / \mathrm{EtOH}=$ 2:1). After equilibration for $30 \mathrm{~min}$, the FITC-Eu-CaCit NP solution was applied on top of the skin and the Franz cell was protected from light and sealed with parafilm for $12 \mathrm{~h}$. Following the incubation for the penetration experiments, the human skin was gently shaken to remove any fluorescent NP residues on the surface of the skin with PBS $\mathrm{pH}$ 7.4. The skin tissues were embedded in an OCT embedding medium and sectioned into $5 \mu \mathrm{m}$ thicknesses by a cryotome. The penetration ability of the NPs was observed using a confocal laser scanning microscope (Zeiss LSM 800, Carl Zeiss, Oberkochen, Germany) at an excitation wavelength of $488 \mathrm{~nm}$.

2.14. Statistical Analysis. All results are mean values \pm standard deviation of three or more experiments. Data were analyzed with the GraphPad Prism 5.0 software (GraphPad Software Inc., San Diego, USA; www.graphpad.com). Statistical data analyses were performed on all data by one-way ANOVA followed by multiparametric Tukey's post hoc test with $p<0.05$ as the minimal level of significance.

\section{RESULTS}

3.1. Characterization of Eu-CaCit NPs. The morphology of Eu-CaCit NPs is shown in Figure 1. The SEM image of EuCaCit NPs showed the spherical NPs with the size in the range of 150-250 nm (Figure 1a). The SEM images of the FITC-
Eu-CaCit NPs revealed the morphology of the NPs as a spherical shape similar to the Eu-CaCit NPs (Figure 1b).

TGAs were conducted to investigate the thermal decomposition behavior of both Eu-CaCit and FITC-Eu-CaCit NPs (Figure 2) together with the derivative thermogravimetric curve. In the case of Eu-CaCit NPs (Figure 2a), the analysis expressed three main regions of weight loss. The first region contains two steps, at $80-120^{\circ} \mathrm{C}$ and at around $120-180^{\circ} \mathrm{C}$, respectively, corresponding to the loss of $\mathrm{H}_{2} \mathrm{O} .{ }^{20}$ The second region, which contributed to $31 \%$ of the weight loss at $300-$ $600{ }^{\circ} \mathrm{C}$, could be assigned to the decomposition of calcium citrate into calcium carbonate $\left(\mathrm{CaCO}_{3}\right)^{20}$ and calcium eugenate that was entrapped in the NPs. The mass loss in the third region was due to the decomposition of $\mathrm{CaCO}_{3}$ to calcium oxide $(\mathrm{CaO}){ }^{20}$ The thermal decomposition and the derivative curve of FITC-Eu-CaCit NPs are also shown in Figure $2 \mathrm{~b}$. The percentage of eugenol in the NPs was calculated to be $5.5 \%$. According to the graph, there was no significant change in the decomposition pattern of the FITCEu-CaCit NPs compared to Eu-CaCit NPs indicating the very thin coating of FITC onto the Eu-CaCit NPs. Furthermore, zeta potential analysis was used to confirm the attachment of FITC on the Eu-CaCit NP surface. The result showed the decreasing of the average zeta potential value of FITC-EuCaCit NPs compared to that of Eu-CaCit NPs (Table S1). Since the FITC molecule has lower polarity compared to 


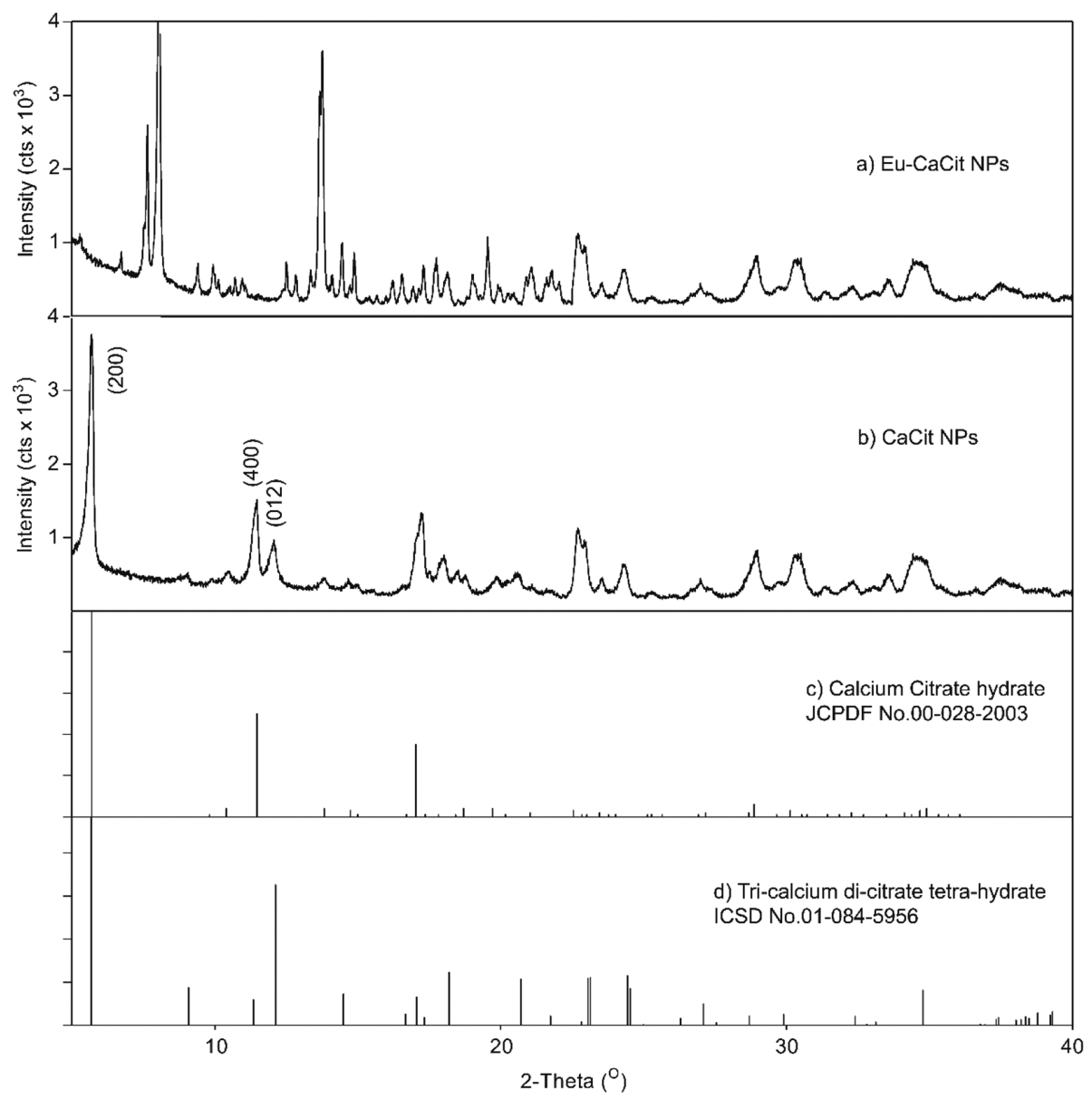

Figure 3. XRD pattern of Eu-CaCit NPs (a), CaCit NPs (b), calcium citrate hydrate (c), and tricalcium dicitrate tetrahydrate (d).
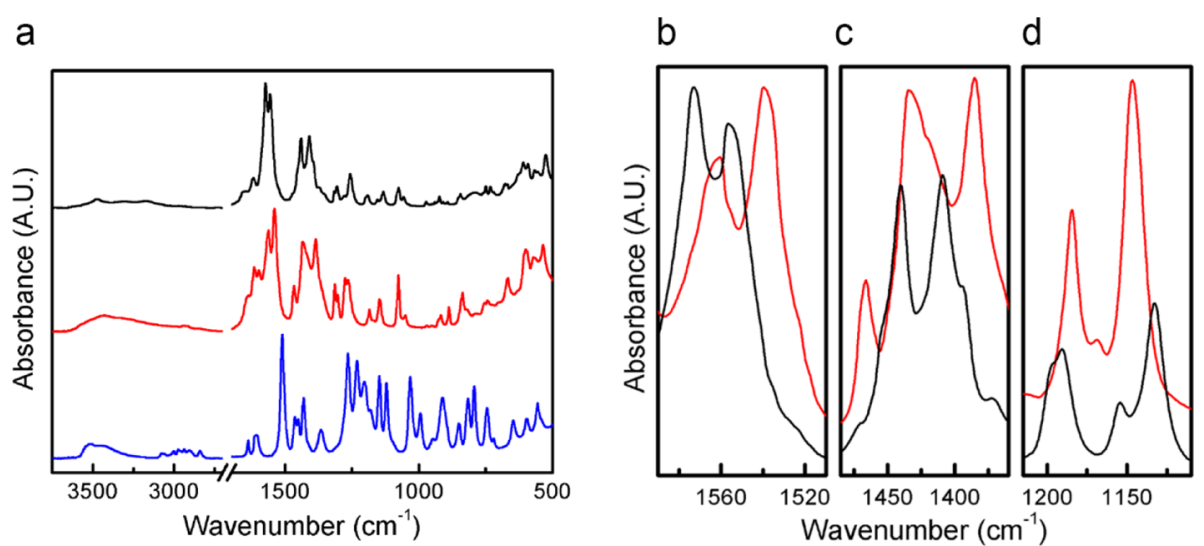

Figure 4. FTIR spectra of eugenol (blue line), CaCit NPs (red line), and Eu-CaCit NPs (black line) in the range of 3750-500 (a), 1590-1510 (b), $1485-1360$ (c), and $1215-1110 \mathrm{~cm}^{-1}(\mathrm{~d})$.

citrate ions, this smaller negative zeta potential value on the NP surface indicated the successful FITC-coating step.

The high crystallinity of Eu-CaCit NPs was observed as shown in Figure 3a. The XRD pattern of the sample exhibited the appearance of new peaks and disappearance of characteristic peaks of standard CaCit NPs (Figure 3b), calcium citrate hydrate (Figure 3c), and tricalcium dicitrate tetrahydrate (Figure 3d). The 200, 400, and 012 faces, characteristic peaks of CaCit NPs, are not observed. On the other hand, the intense peaks at diffraction angles $2 \theta$ equal to $7.6,8.0$, and $13.7^{\circ}$ were observed. These results implied that eugenol could preferably bind onto some faces of the CaCit NPs.

From Figure 4a, the FTIR spectrum of eugenol shows characteristic peaks at $1605,1430,994$, and $793 \mathrm{~cm}^{-1}$, which attribute to the vibration modes of $\mathrm{C}=\mathrm{C}$ aromatic stretching, $\mathrm{CH}_{2}$ deformation, $\mathrm{CH}$ out-of-plane bending, and ring deformation, respectively. ${ }^{21}$ Characteristic peaks of $\mathrm{CaCit}$ NPs can also be observed at 1561 and $1540 \mathrm{~cm}^{-1}$ arising from asymmetric carboxylate stretching vibration, while the characteristic peaks of symmetric carboxylate stretching vibration can be observed at 1434 and $1385 \mathrm{~cm}^{-1} .{ }^{20}$ Moreover, 

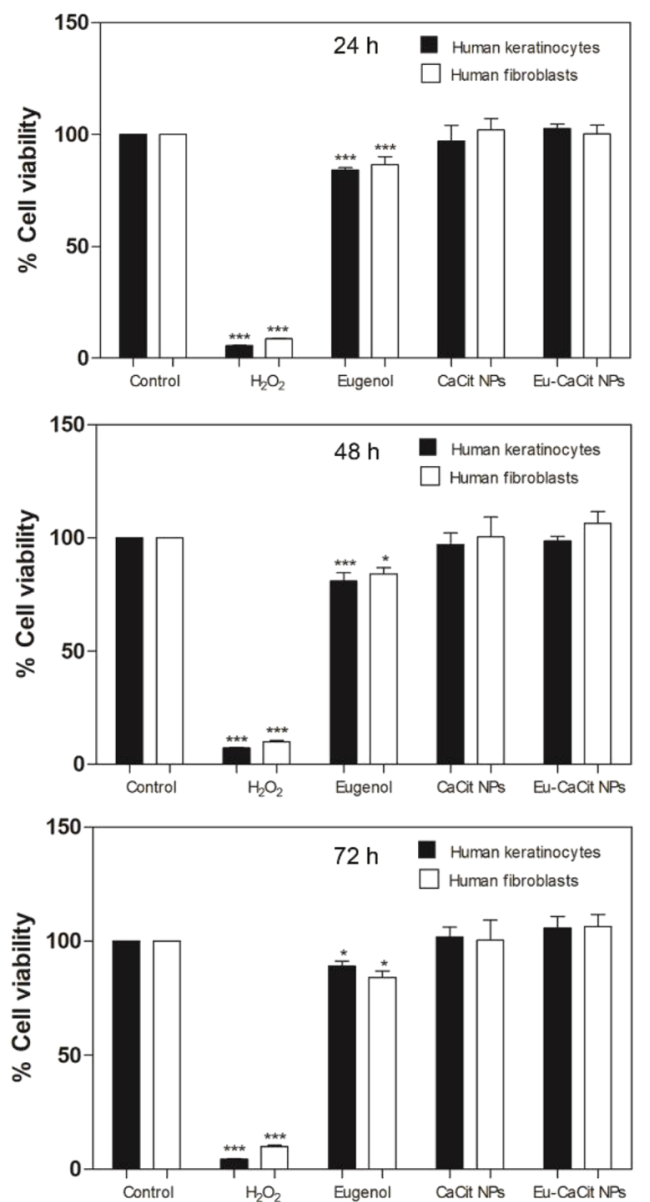

b

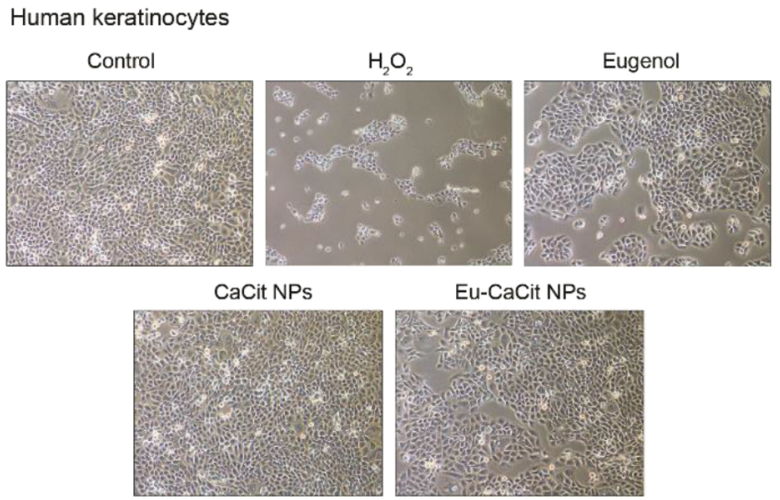

Human fibroblasts
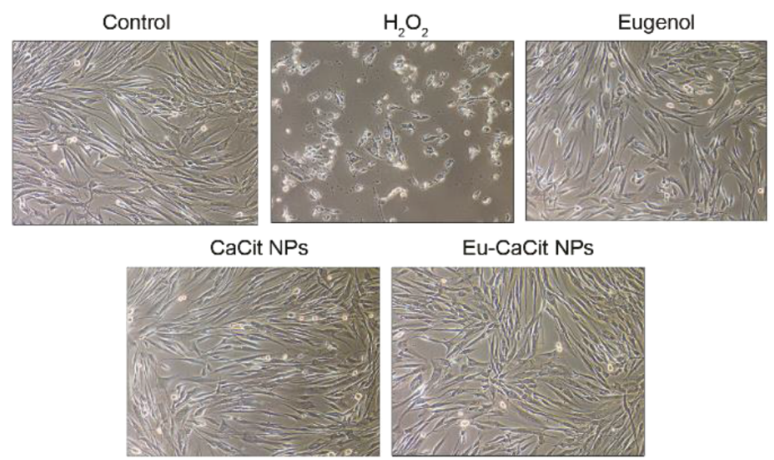

Eu-CaCit NPs

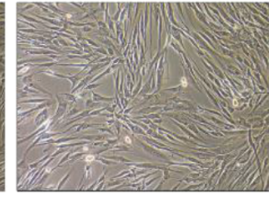

Figure 5. Cytotoxicity of Eu-CaCit NPs in human keratinocytes and fibroblasts. Cell viability of human cells after incubation with eugenol, CaCit $\mathrm{NPs}$, and Eu-CaCit NPs for 24, 48, and $72 \mathrm{~h}$ (a). Cell morphology of human keratinocytes and fibroblasts incubated with eugenol, CaCit NPs, and Eu-CaCit NPs for $24 \mathrm{~h}(\mathrm{~b})$. Data are shown as mean $\pm \mathrm{SD}$, derived from three repeats where $*=p<0.05$, $* * *=p<0.001$ compared to the control group.

peaks at 1185 and $1146 \mathrm{~cm}^{-1}$ indicate $\mathrm{C}-\mathrm{O}$ stretching vibration of citrate. ${ }^{22}$ Detailed band assignments for $\mathrm{CaCit}$ NPs and eugenol are listed in Table S2. Figure $4 b-d$ shows FTIR spectra of CaCit NPs and Eu-CaCit NPs in the range of $1590-1510,1485-1360$, and $1215-1110 \mathrm{~cm}^{-1}$. The peaks of citrate at $1561,1540,1434$, and $1385 \mathrm{~cm}^{-1}$ in the sample of $\mathrm{CaCit}$ NPs shifted to higher wavenumbers at 1573, 1556, 1440, and $1409 \mathrm{~cm}^{-1}$ in the sample of Eu-CaCit NPs, respectively. Moreover, the peaks of citrate at 1185 and $1146 \mathrm{~cm}^{-1}$ shifted to 1191 and $1133 \mathrm{~cm}^{-1}$, respectively.

3.2. Cytotoxicity of Eu-CaCit NPs. In order to sufficiently study the potential for Eu-CaCit NPs serving as a new drug, the cytotoxicity of Eu-CaCit NPs was first evaluated in human keratinocytes and fibroblasts with the use of the PrestoBlue cell viability reagent. The cell morphology was observed after treatment for $24 \mathrm{~h}$. The cytotoxicity effect of Eu-CaCit NPs is shown in Figure 5. The treatment of eugenol showed a clear dose-dependent cytotoxicity in human keratinocytes and fibroblasts (Figure S1). There was no significant decrease in cell viability when cells were treated with $\mathrm{CaCit} \mathrm{NPs}$ and $\mathrm{Eu}$ CaCit NPs (Figure 5a). There was no difference in cell morphology between NPs and the control group (Figure 5b). Therefore, CaCit NPs and Eu-CaCit NPs exhibited no obvious cytotoxicity and exhibited excellent biocompatibility for skin cells.

3.3. Cellular Uptake of Eu-CaCit NPs. The cellular uptake ability of Eu-CaCit NPs was conducted in human keratinocytes and fibroblasts at $24 \mathrm{~h}$ post incubation. The EuCaCit NPs were modified with FITC in order to visualize their intracellular delivery into the cells. The uptake of FITC-EuCaCit NPs was observed in human keratinocytes (Figure 6a) and fibroblasts (Figure 6b). The fluorescence intensity suggested that there was a greater amount of Eu-CaCit NPs in the cellular uptake at $24 \mathrm{~h}$.

3.4. Drug Release Profiles of Eugenol. The release of eugenol from Eu-CaCit NPs was investigated at different $\mathrm{pH}$ (4.8, 5.5, and 7.4) conditions. Our results showed that the eugenol release rate was increased along with the decrease of the $\mathrm{pH}$ value. As illustrated in Figure 7, the eugenol release behavior was observed with initial bursting of NPs in $15 \mathrm{~min}$ followed by slow and sustained release in $90 \mathrm{~min}$. Eu-CaCit NPs were more easily released under acidic conditions ( $\mathrm{pH} 4.8$ and 5.5) than under weak basic conditions ( $\mathrm{pH} 7.4)$. It was observed that 94 and $90 \%$ release was obtained in acidic $\mathrm{pH}$ ( $\mathrm{pH} 4.8$ and 5.5) as opposed to 59\% in neutral $\mathrm{pH}$. The cumulative release of eugenol showed only a slight increase at 
a

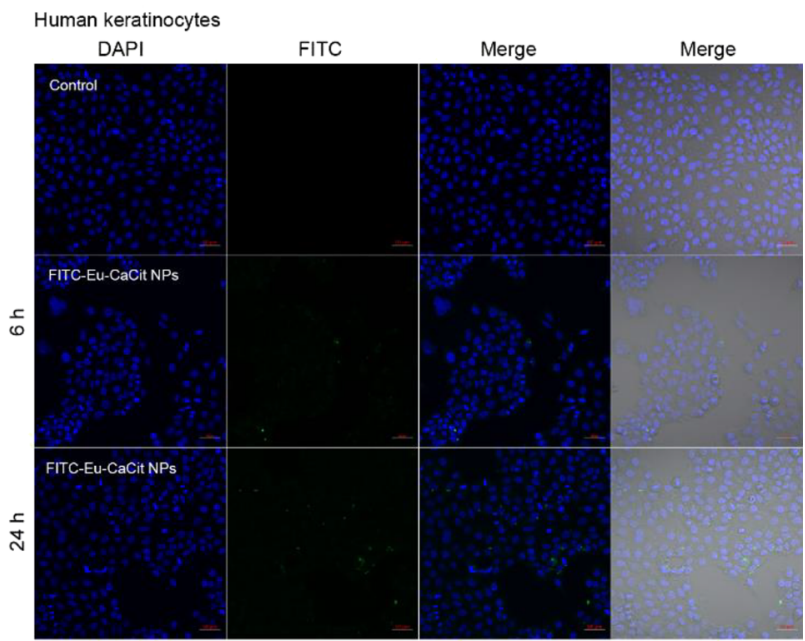

b

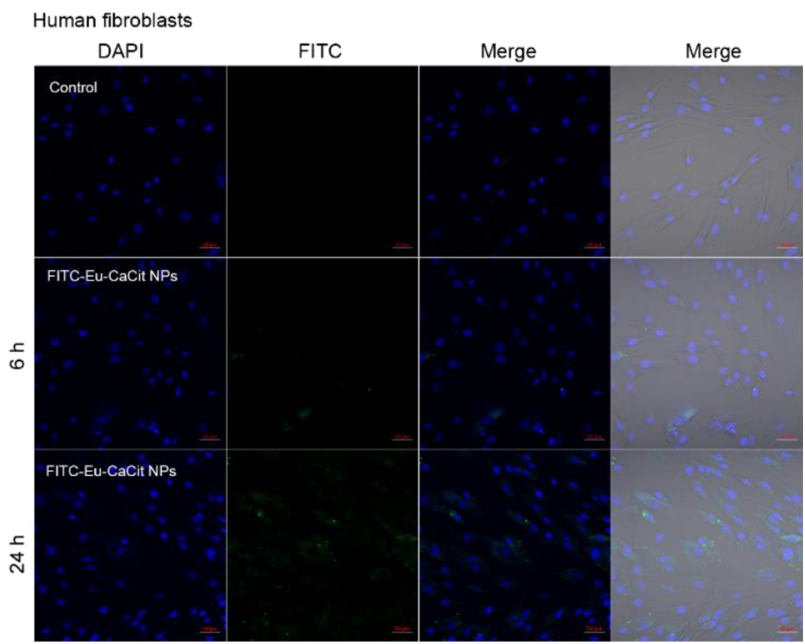

Figure 6. Cellular uptake results of FITC-Eu-CaCit NPs in human keratinocytes and fibroblasts. Cells were incubated with FITC-Eu$\mathrm{CaCit}$ NPs in human keratinocytes (a) and fibroblasts (b) at $37{ }^{\circ} \mathrm{C}$ for $24 \mathrm{~h}$, followed by DAPI (4',6-diamidino-2-phenylindole) staining and rinsing with phosphate buffered saline. Intracellular fluorescence was detected by a confocal laser scanning microscope.

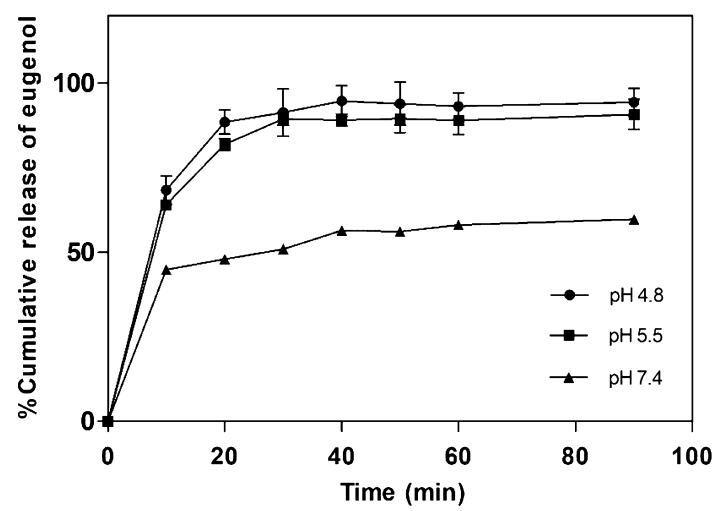

Figure 7. Drug release profile of Eu-CaCit NPs under different $\mathrm{pH}$

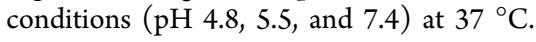

$\mathrm{pH} 4.8$, when compared with the cumulative release of eugenol at $\mathrm{pH} 5.5$.
3.5. Penetration Ability of Eu-CaCit NPs into the Human Skin. To investigate the penetration ability of the EuCaCit NPs, they were labeled with FITC to monitor tracking NPs in the human skin. The penetration of FITC-Eu-CaCit NPs into the intact skin ex vivo following $12 \mathrm{~h}$ exposure is shown in Figure 8. Fluorescence was observed in the stratum corneum and viable epidermis (Figure $8 b$ ) when compared with the nontreated skin (Figure 8a). Moreover, the Eu-CaCit NPs were able to penetrate the dermis and also retained in the upper skin layers. NPs were able to penetrate the dermis, while the NPs were retained in the upper skin layers. Therefore, this result demonstrated the promising application of the Eu-CaCit NPs as a topical drug delivery platform using CaCit NPs as a drug delivery carrier.

\section{DISCUSSION}

Eugenol (4-allyl-2-methoxy phenol), is a phenolic compound from the class of phenylpropanoids and the main compound occurring in cloves associated with their health benefits. ${ }^{23-25}$ It has been used as a flavoring agent for foods and cosmetics and as a preservative in food industry. In addition, there are many biological effects such as antioxidant, anti-inflammatory, antianaphylactic effects, and antimicrobial properties. ${ }^{26,27}$ Interestingly, eugenol is also known as a local analgesic agent in dentistry, which is associated with the activation of specialized peripheral sensory neurons. The subcutaneous injection of eugenol inhibits pain behavior through the blockade of action potential conduction in the peripheral nerve in the orofacial area. It also reversibly inhibits action potential and VGSC in $\mathrm{IB}_{4+}$ and $\mathrm{TRPV}_{1+}$ nociceptive trigeminal ganglion neurons. ${ }^{10}$ These effects are reminiscent of the effects of local anesthetics such as lidocaine. ${ }^{28}$ These results suggest that eugenol can be applied for other pathological pain conditions with the local anesthetic effect. However, eugenol has been reported to induce skin irritation by perturbation of the stratum corneum. ${ }^{29}$ Consequently, delivery of eugenol using $\mathrm{CaCit} \mathrm{NPs}$ was evaluated in the human skin, compromising the nontoxic carrier.

In this study, Eu-CaCit NPs were successfully synthesized using the coprecipitation reaction of calcium and citrate ions in the presence of eugenol. The optimum condition for CaCit NP synthesis has been studied in our research group, which suggests that the mole ratio between calcium and citrate ions should be 1.0:1.3 to make well size-distributed and spherical NPs. Molecular eugenol was treated with $\mathrm{NaOH}$ to deprotonate the phenolic proton to create phenolate anions for increasing the binding constant with calcium ions by electrostatic interaction. After completion of calcium ion addition, a white milky colloid was obtained within $10 \mathrm{~min}$, indicating the formation of CaCit NPs. The particle size was observed by SEM with the size in the range of $150-250 \mathrm{~nm}$. This result is in agreement with the previous study describing that the excess citrate ion can act as a stabilizer in the formation of NPs by increasing the electrostatic repulsion on the particle surface due to a high negative charge. ${ }^{30,31}$

We then investigated the potential application of Eu-CaCit NPs as molecular carriers for drug delivery by introducing FITC as a fluorescent probe onto the Eu-CaCit NP surface. ${ }^{32}$ This surface modification could be achieved conveniently ${ }^{19}$ by mixing the Eu-CaCit NP colloidal mixture with the anionic FITC generated under basic conditions for $1 \mathrm{~h}$, leading to the formation of FITC-Eu-CaCit NPs. 

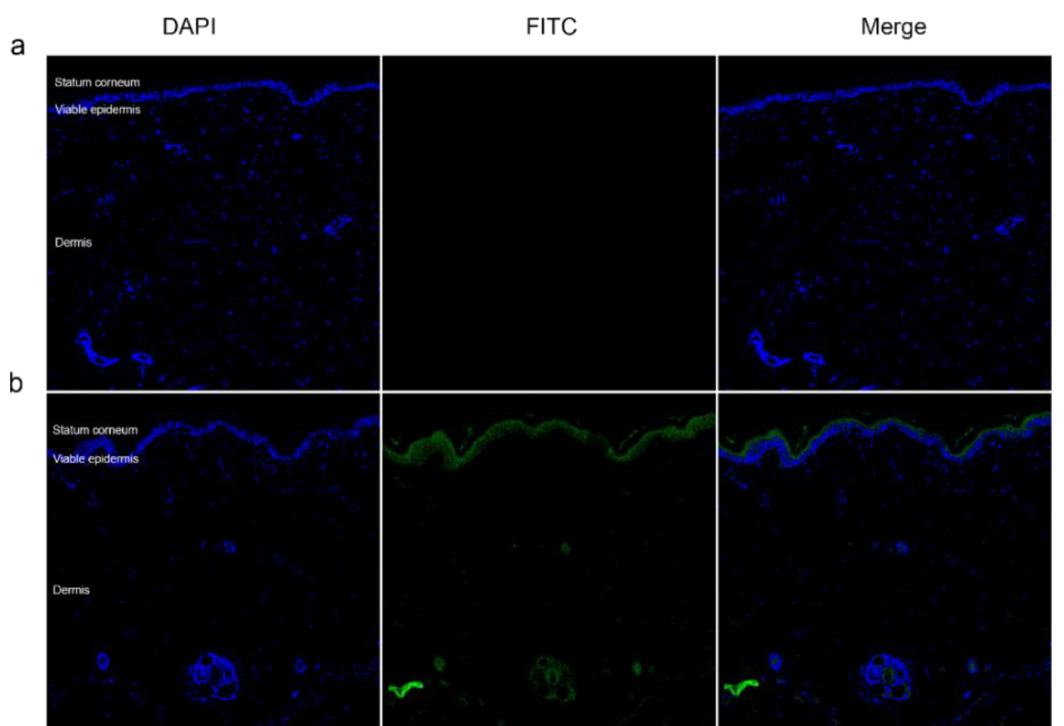

Figure 8. Confocal laser scanning microscopy images of human skin samples from the skin penetration study. The FITC-Eu-CaCit NPs (green fluorescence) were applied ex vivo on the human skin for $12 \mathrm{~h}$. Histological sections are shown using confocal microscopy control (a) and FITCEu-CaCit NPs (b) at an excitation wavelength of $488 \mathrm{~nm}$, with nuclei of cells (blue) stained with DAPI.

The thermal decomposition behaviors of both Eu-CaCit and FITC-Eu-CaCit NPs expressed three main regions of weight loss. The first region contains two steps, the first step at 80$120{ }^{\circ} \mathrm{C}$ could be corresponded to the loss of surface-adsorbed water molecules and a part of the crystal water molecules. ${ }^{20,33}$ The second step at around $120-180{ }^{\circ} \mathrm{C}$ could be due to the subsequent removal of others two water molecules in the $\mathrm{Ca}_{3}\left(\mathrm{C}_{6} \mathrm{H}_{5} \mathrm{O}_{7}\right)_{2} \cdot 2 \mathrm{H}_{2} \mathrm{O}$ compound as shown in the following equations:

$$
\begin{aligned}
& {\left[\mathrm{Ca}_{3}\left(\mathrm{C}_{6} \mathrm{H}_{5} \mathrm{O}_{7}\right)_{2}\left(\mathrm{H}_{2} \mathrm{O}\right)_{2}\right] \cdot 2 \mathrm{H}_{2} \mathrm{O}(\mathrm{s})} \\
& \quad \rightarrow \mathrm{Ca}_{3}\left(\mathrm{C}_{6} \mathrm{H}_{5} \mathrm{O}_{7}\right)_{2} \cdot 2 \mathrm{H}_{2} \mathrm{O}(\mathrm{s})+2 \mathrm{H}_{2} \mathrm{O}(\mathrm{g}) \\
& \mathrm{Ca}_{3}\left(\mathrm{C}_{6} \mathrm{H}_{5} \mathrm{O}_{7}\right)_{2}\left(\mathrm{H}_{2} \mathrm{O}\right)_{2}(\mathrm{~s}) \\
& \quad \rightarrow \mathrm{Ca}_{3}\left(\mathrm{C}_{6} \mathrm{H}_{5} \mathrm{O}_{7}\right)_{2}(\mathrm{~s})+2 \mathrm{H}_{2} \mathrm{O}(\mathrm{g})
\end{aligned}
$$

The second region, ${ }^{20}$ which contributed to $31 \%$ of the weight loss, appears at $300-600{ }^{\circ} \mathrm{C}$ and could be assigned to the decomposition of calcium citrate into calcium carbonate $\left(\mathrm{CaCO}_{3}\right)$ as shown in the equation:

$$
\mathrm{Ca}_{3}\left(\mathrm{C}_{6} \mathrm{H}_{5} \mathrm{O}_{7}\right)_{2}(\mathrm{~s}) \rightarrow 3 \mathrm{CaCO}_{3}(\mathrm{~s})+5 \mathrm{H}_{2} \mathrm{O}(\mathrm{g})+9 \mathrm{C}(\mathrm{s})
$$

The decomposition in this region was used to investigate the amount of eugenol content in the Eu-CaCit NPs. According to the literature, ${ }^{34}$ the percent weight loss from the above equation was normally equal to $25.5 \%$, and the decomposition temperature of calcium alkoxide was in the range of 350-450 ${ }^{\circ} \mathrm{C} .{ }^{35}$ Therefore, the difference of $5.5 \%$ of the weight loss in this study could be referred to the amount of eugenol in the NPs.

FTIR technique was exploited to determine intermolecular interactions between eugenol and CaCit NPs in Eu-CaCit NPs. As seen from Figure 4a, the FTIR spectrum of Eu-CaCit NPs is mainly dominated by that of CaCit NPs suggesting that there is a small amount of eugenol in Eu-CaCit NPs. However, the intermolecular interactions between eugenol and CaCit NPs can still be analyzed from peak shifts of citrate, as shown in Figure $4 \mathrm{~b}-\mathrm{d}$; the peaks of citrate at 1561, 1540, 1434, and $1385 \mathrm{~cm}^{-1}$ in the sample of CaCit NPs shift to higher wavenumbers at $1573,1556,1440$, and $1409 \mathrm{~cm}^{-1}$ in the sample of Eu-CaCit NPs, respectively. This indicates the strengthening of bonds in carboxylate groups, suggesting the interactions between carboxylate groups in citrate and hydroxyl or ether groups in eugenol. Moreover, the peaks of citrate at 1185 and $1146 \mathrm{~cm}^{-1}$ shift to 1191 and $1133 \mathrm{~cm}^{-1}$, respectively, implying that oxygen in the $\mathrm{C}-\mathrm{O}$ bond interacts with a hydroxyl or ether group in eugenol. By these results, it can be concluded that there are intermolecular interactions binding citrate and eugenol together in Eu-CaCit NPs.

The cytotoxicity percentages of eugenol at increased concentrations on human keratinocytes and fibroblasts were evaluated in human skin cells (Figure S1). On the other hand, the viability of all the NP-treated groups was not significantly different from untreated cells in either viability assay throughout the $72 \mathrm{~h}$ of treatment. In our previous study, CaCit NPs did not show any meaningful toxicity in human keratinocytes at concentrations of $0.3,0.6$, and $1.2 \mathrm{~g} / \mathrm{L} .{ }^{19} \mathrm{We}$ performed a cytotoxicity assay in order to determine nontoxic physiological concentrations of CaCit NPs and Eu-CaCit NPs in human keratinocytes and fibroblasts at concentrations of 0.3 , 0.6 , and $1.2 \mathrm{~g} / \mathrm{L}$. The results did not show any remarkable changes on the cell viability when compared with the control (Figures S2 and S3). Therefore, the nontoxic concentration $(1.2 \mathrm{~g} / \mathrm{L})$ of Eu-CaCit NPs was selected for use in further experiments. $\mathrm{CaCit}$ has been proved to be a biocompatible material because the citrate ion itself is an intermediate in the citric acid cycle. ${ }^{20,36}$ It has been shown that CaCit NPs are beneficial as drug carriers to transport eugenol into the skin without toxicity effects.

The drug release behavior of Eu-CaCit NPs is important to evaluate when using NPs in the drug delivery systems. The $\mathrm{pH}$ change occurs at many specific or physiological sites in the body, for example, the skin environment is more acidic $(\mathrm{pH}$ 5.5) than blood and normal tissues ( $\mathrm{pH} 7.4) .^{37-39} \mathrm{CaCO}_{3} \mathrm{NPs}$ showed a slower release of drugs in normal physiological $\mathrm{pH}$ (7.4) compared to that in an acidic environment. ${ }^{40}$ The release profile of $\mathrm{Eu}$ from $\mathrm{CaCit}$ NPs was studied under various conditions. The acidic $\mathrm{pH}(\mathrm{pH} 4.8$ and 5.5) showed control release properties, which indicates cumulative release profiles 
of eugenol from Eu-CaCit NPs at an initial burst release. Release kinetic modeling of Eu from our study was analyzed using the zero-order, first-order, Higuchi, and KorsmeyerPeppas model. ${ }^{41}$ The result showed that the Higuchi model fitted well and is suitable for the release of $\mathrm{Eu}$ in the acidic environment. According to the Higuchi model, which refers to the Fickian diffusion mechanism of drug release from $\mathrm{CaCit}$ NPs, the $n$ value is 0.5 . It indicated the limited diffusion kinetics of the $\mathrm{Eu}$ release mechanism. Our results suggested that the $\mathrm{pH}$-sensitive properties of Eu-CaCit NPs in acidic $\mathrm{pH}$ makes them a suitable nanocarrier for topical treatment owing to their more acidic nature.

The penetration ability of FITC-Eu-CaCit NPs was observed by confocal microscopy after incubation time at 12 h. The results showed that FITC-Eu-CaCit NPs penetrate across the stratum corneum into the dermis. It is observed that $\mathrm{Eu}-\mathrm{CaCit}$ NPs penetrate through the human skin through skin appendages such as hair follicles and cutaneous glands. Small molecules (both lipophilic and hydrophilic) can penetrate the skin through sweat glands and hair shafts. ${ }^{14}$ However, a high accumulation of Eu-CaCit NPs was found in the stratum corneum. This effect may serve as the drug reservoir for extended release into the viable epidermis over hours. The NPs may increase the penetration ability of a drug to deeper layers of the skin due to their outstanding properties. ${ }^{42}$ The use of nanostructures has gained increasing opportunities to deliver the therapeutic agent to the skin without compromising its barrier function. ${ }^{43}$ The development of $\mathrm{Eu}-\mathrm{CaCit} \mathrm{NPs}$ has become an alternative agent for local anesthetic. It is an attractive hypothesis that Eu-CaCit NPs retain drug ability for a longer time by increasing skin penetration without skin irritation. The skin irritation potential of Eu-CaCit NPs will be performed by comparison with $\mathrm{Eu}$ and conventional local anesthetics. However, providing this hypothesis will require a better understanding of Eu-CaCit NPs such as stability, interactions with the skin components, and the transport mechanism. Moreover, further work is required to investigate the antinociceptive effects of Eu-CaCit NPs compared to eugenol-induced antinociception in in vitro and in vivo studies.

\section{CONCLUSIONS}

Novel Eu-CaCit NPs were successfully prepared to facilitate the delivery of eugenol into the human intact skin. The biocompatible carrier promises to overcome the skin irritation and other side effects. More importantly, the Eu-CaCit NPs might be notional candidates as a local anesthetic to improve patient compliance, safety, and efficacy of drugs. To our knowledge, this is the first study that investigated the use of $\mathrm{Eu}-\mathrm{CaCit} \mathrm{NPs}$ as a new drug for topical anesthetic application.

\section{ASSOCIATED CONTENT}

\section{(s) Supporting Information}

The Supporting Information is available free of charge at https://pubs.acs.org/doi/10.1021/acsomega.1c03831.

Cytotoxicity evaluation, zeta potential, and FTIR band assignments for CaCit NPs and eugenol (PDF)

\section{AUTHOR INFORMATION}

\section{Corresponding Authors}

Rojrit Rojanathanes - Center of Excellence in Materials and Bio-Interfaces, Faculty of Science, Chulalongkorn University,
Bangkok 10330, Thailand; 이이이.org/0000-0002-05063583; Email: rojrit@hotmail.com

Pornanong Aramwit - Department of Pharmacy Practice, Faculty of Pharmaceutical Sciences and Center of Excellence in Bioactive Resources for Innovative Clinical Applications, Chulalongkorn University, Bangkok 10330, Thailand; The Academy of Science, The Royal Society of Thailand, Bangkok 10330, Thailand; Email: aramwit@gmail.com

\section{Authors}

Sarocha Cherdchom - Department of Pharmacy Practice, Faculty of Pharmaceutical Sciences, Chulalongkorn University, Bangkok 10330, Thailand

Wittawat Keawsongsaeng - Department of Chemistry, Faculty of Science, Chulalongkorn University, Bangkok 10330, Thailand

Wanida Buasorn - Department of Anatomy, Faculty of Medicine, Chulalongkorn University, Bangkok 10330, Thailand

Natchanon Rimsueb - Department of Chemistry, Faculty of Science, Chulalongkorn University, Bangkok 10330, Thailand

Prompong Pienpinijtham - Sensor Research Unit (SRU) and National Nanotechnology Center of Advanced Structural and Functional Nanomaterials, Department of Chemistry, Faculty of Science, Chulalongkorn University, Bangkok 10330,

Thailand; 이이.org/0000-0002-2956-9132

Amornpun Sereemaspun - Nanomedicine Research Unit, Department of Anatomy, Faculty of Medicine, Chulalongkorn University, Bangkok 10330, Thailand

Complete contact information is available at:

https://pubs.acs.org/10.1021/acsomega.1c03831

\section{Author Contributions}

S.C. carried out the experiment and wrote the manuscript with input from all authors. W.K. carried out the preparation and characterization of nanoparticles. W.B. conducted histological experiments. N.R. and P.P. carried out the preparation and characterization of nanoparticles. A.S. reviewed and revised the draft. R.R. and P.A. contributed to the design and implementation of the research and contributed to the final version of the manuscript. The manuscript was written through contributions of all authors. All authors have given approval to the final version of the manuscript. R.R. and P.A. authors contributed equally.

\section{Funding}

This work was supported by the Ratchadaphiseksomphot Endowment Fund [Project Code 762007], Chulalongkorn University, and the National Research Council of Thailand, Thailand.

\section{Notes}

The authors declare no competing financial interest.

\section{ACKNOWLEDGMENTS}

This work was supported by the Ratchadaphiseksomphot Endowment Fund [Project Code 762007], Chulalongkorn University, and the National Research Council of Thailand, Thailand. The authors are also grateful to Dr. Jiraroch Meevassana for providing the primary human fibroblasts. The authors specially thank Prof. Suwabun Chirachanchai's laboratory at The Petroleum and Petrochemical College and 
Mr. Vitavat Aksornkitti for their assistance throughout this project.

\section{ABBREVIATIONS}

$\mathrm{Eu}$, eugenol; NPs, nanoparticles; Eu-CaCit NPs, eugenolembedded calcium citrate nanoparticles; $\mathrm{CaCit} \mathrm{NPs}$, calcium citrate nanoparticles; $\mathrm{NaOH}$, sodium hydroxide; FITC, fluorescein 5(6)-isothiocyanate isomer I; DI water, deionized water; SEM, scanning electron microscopy; TGA, thermogravimetric analysis; XRD, X-ray powder diffraction; FTIR, Fourier transform infrared spectroscopy

\section{REFERENCES}

(1) Bhusal, P.; Harrison, J.; Sharma, M.; Jones, D. S.; Hill, A. G.; Svirskis, D. Controlled release drug delivery systems to improve postoperative pharmacotherapy. Drug Deliv. Transl. Res. 2016, 6, 441451.

(2) Griggs, C.; Goverman, J.; Bittner, E. A.; Levi, B. Sedation and Pain Management in Burn Patients. Clin. Plast. Surg. 2017, 44, 535540.

(3) Katz, N. The impact of pain management on quality of life. J. Pain Symptom Manage. 2002, 24, S38-S47.

(4) Cheung, H. M.; Lee, S. M.; MacLeod, B. A.; Ries, C. R.; Schwarz, S. K. A comparison of the systemic toxicity of lidocaine versus its quaternary derivative QX-314 in mice. Can. J. Anaesth. 2011, $58,443-450$.

(5) Kandil, E.; Melikman, E.; Adinoff, B. Lidocaine Infusion: A Promising Therapeutic Approach for Chronic Pain. J. Anesth. Clin. Res. 2017, 08, 697.

(6) Scott, D. B.; Lee, A.; Fagan, D.; Bowler, G. M. R.; Bloomfield, P.; Lundh, R. Acute toxicity of ropivacaine compared with that of bupivacaine. Anesth. Analg. 1989, 69, 563-569.

(7) Ito, M.; Murakami, K.; Yoshino, M. Antioxidant action of eugenol compounds: role of metal ion in the inhibition of lipid peroxidation. Food Chem. Toxicol. 2005, 43, 461-466.

(8) Prasad, S. N.; Muralidhara. Neuroprotective efficacy of eugenol and isoeugenol in acrylamide-induced neuropathy in rats: behavioral and biochemical evidence. Neurochem. Res. 2013, 38, 330-345.

(9) Yogalakshmi, B.; Viswanathan, P.; Anuradha, C. V. Investigation of antioxidant, anti-inflammatory and DNA-protective properties of eugenol in thioacetamide-induced liver injury in rats. Toxicology 2010, 268, 204-212.

(10) Park, C. K.; Kim, K.; Jung, S. J.; Kim, M. J.; Ahn, D. K.; Hong, S. D.; Kim, J. S.; Oh, S. B. Molecular mechanism for local anesthetic action of eugenol in the rat trigeminal system. Pain 2009, 144, 84-94.

(11) Shah, B.; Davidson, P. M.; Zhong, Q. Nanodispersed eugenol has improved antimicrobial activity against Escherichia coli O157:H7 and Listeria monocytogenes in bovine milk. Int. J. Food Microbiol. 2013, 161, 53-59.

(12) Tammannavar, P.; Pushpalatha, C.; Jain, S.; Sowmya, S. V. An unexpected positive hypersensitive reaction to eugenol. BMJ Case Rep. 2013, 2013, No. bcr2013009464.

(13) van der Maaden, K.; Jiskoot, W.; Bouwstra, J. Microneedle technologies for (trans)dermal drug and vaccine delivery. J. Controlled Release 2012, 161, 645-655.

(14) Goyal, R.; Macri, L. K.; Kaplan, H. M.; Kohn, J. Nanoparticles and nanofibers for topical drug delivery. J. Controlled Release 2016, 240, 77-92.

(15) Palmer, B. C.; DeLouise, L. A. Nanoparticle-Enabled Transdermal Drug Delivery Systems for Enhanced Dose Control and Tissue Targeting. Molecules 2016, 21, 1719.

(16) De Jong, W. H.; Borm, P. J. Drug delivery and nanoparticles:applications and hazards. Int. J. Nanomed. 2008, 3, 133-149.

(17) Ding, Y.; Shen, S. Z.; Sun, H.; Sun, K.; Liu, F.; Qi, Y.; Yan, J. Design and construction of polymerized-chitosan coated $\mathrm{Fe} 3 \mathrm{O} 4$ magnetic nanoparticles and its application for hydrophobic drug delivery. Mater. Sci. Eng. C Mater. Biol. Appl. 2015, 48, 487-498.
(18) Ferris, D. P.; Lu, J.; Gothard, C.; Yanes, R.; Thomas, C. R.; Olsen, J. C.; Stoddart, J. F.; Tamanoi, F.; Zink, J. I. Synthesis of biomolecule-modified mesoporous silica nanoparticles for targeted hydrophobic drug delivery to cancer cells. Small 2011, 7, 1816-1826.

(19) Rimsueb, N.; Cherdchom, S.; Aksornkitti, V.; Khotavivattana, T.; Sereemaspun, A.; Rojanathanes, R. Feeding Cells with a Novel "Trojan" Carrier: Citrate Nanoparticles. ACS Omega 2020, 5, 74187423.

(20) Li, J.; Liu, Y.; Gao, Y.; Zhong, L.; Zou, Q.; Lai, X. Preparation and properties of calcium citrate nanosheets for bone graft substitute. Bioengineered 2016, 7, 376-381.

(21) Wang, L.-H.; Sung, W.-C. Rapid evaluation and quantitative analysis of eugenol derivatives in essential oils and cosmetic formulations on human skin using attenuated total reflectanceinfrared spectroscopy. Spectroscopy 2011, 26, 43-52.

(22) Socrates, G., Infrared and Raman Characteristic Group Frequencies: Tables and Charts. 3 ed.; John Wiley \& Sons, Inc.: New York City, United States, 2004.

(23) Atsumi, T.; Fujisawa, S.; Tonosaki, K. A comparative study of the antioxidant/prooxidant activities of eugenol and isoeugenol with various concentrations and oxidation conditions. Toxicol. In Vitro 2005, 19, 1025-1033.

(24) Barboza, J. N.; da Silva Maia Bezerra Filho, C.; Silva, R. O.; Medeiros, J. V. R.; de Sousa, D. P. An Overview on the Antiinflammatory Potential and Antioxidant Profile of Eugenol. Oxid. Med. Cell. Longevity 2018, 2018, No. 3957262.

(25) Bezerra, D. P.; Militão, G.; de Morais, M. C.; de Sousa, D. P. The Dual Antioxidant/Prooxidant Effect of Eugenol and Its Action in Cancer Development and Treatment. Nutrients 2017, 9, 1367.

(26) Kim, H. M.; Lee, E. H.; Kim, C. Y.; Chung, J. G.; Kim, S. H.; Lim, J. P.; Shin, T. Y. Antianaphylactic properties of eugenol. Pharmacol. Res. 1997, 36, 475-480.

(27) Markowitz, K.; Moynihan, M.; Liu, M.; Kim, S. Biologic properties of eugenol and zinc oxide-eugenol. A clinically oriented review. Oral Surg. Oral Med. Oral Pathol. 1992, 73, 729-737.

(28) Leffler, A.; Fischer, M. J.; Rehner, D.; Kienel, S.; Kistner, K.; Sauer, S. K.; Gavva, N. R.; Reeh, P. W.; Nau, C. The vanilloid receptor TRPV1 is activated and sensitized by local anesthetics in rodent sensory neurons. J. Clin. Invest. 2008, 118, 763-776.

(29) El Khayat, N. W.; Donia, A. A.; Mady, O. Y.; El Maghraby, G. M. Optimization of eugenol microemulsion for transdermal delivery of indomethacin. J. Drug Deliv. Sci. Technol. 2018, 48, 311-318.

(30) Martínez-Casado, F. J.; Iafisco, M.; Delgado-López, J. M.; Martínez-Benito, C.; Ruiz-Pérez, C.; Colangelo, D.; Oltolina, F.; Prat, M.; Gómez-Morales, J. Bioinspired Citrate-Apatite Nanocrystals Doped with Divalent Transition Metal Ions. Cryst. Growth Des. 2016, $16,145-153$.

(31) Iafisco, M.; Ramírez-Rodríguez, G. B.; Sakhno, Y.; Tampieri, A.; Martra, G.; Gómez-Morales, J.; Delgado-López, J. M. The growth mechanism of apatite nanocrystals assisted by citrate: relevance to bone biomineralization. CrystEngComm 2015, 17, 507-511.

(32) Riggs, J. L.; Seiwald, R. J.; Burckhalter, J. H.; Downs, C. M.; Metcalf, T. G. Isothiocyanate compounds as fluorescent labeling agents for immune serum. Am. J. Pathol. 1958, 34, 1081-1097.

(33) Herdtweck, E.; Kornprobst, T.; Sieber, R.; Straver, L.; Plank, J. Crystal Structure, Synthesis, and Properties of tri-Calcium di-Citrate tetra-Hydrate [Ca3(C6H5O7)2(H2O)2]-2H2O. Z. Anorg. Allg. Chem. 2011, 637, 655-659.

(34) Mansour, S. A. A. Thermal decomposition of calcium citrate tetrahydrate. Thermochim. Acta 1994, 233, 243-256.

(35) Teo, S. H.; Taufiq-Yap, Y. H.; Rashid, U.; Islam, A. Hydrothermal effect on synthesis, characterization and catalytic properties of calcium methoxide for biodiesel production from crude Jatropha curcas. RSC Adv. 2015, 5, 4266-4276.

(36) Huang, S.; Chen, J. C.; Hsu, C. W.; Chang, W. H. Effects of nano calcium carbonate and nano calcium citrate on toxicity in ICR mice and on bone mineral density in an ovariectomized mice model. Nanotechnology 2009, 20, No. 375102. 
(37) Li, B.; Shan, M.; Di, X.; Gong, C.; Zhang, L.; Wang, Y.; Wu, G. A dual $\mathrm{pH}$ - and reduction-responsive anticancer drug delivery system based on PEG-SS-poly(amino acid) block copolymer. RSC Adv. 2017, 7, 30242-30249.

(38) Nováčková, A.; Sagrafena, I.; Pullmannová, P.; Paraskevopoulos, G.; Dwivedi, A.; Mazumder, A.; Rǔžičková, K.; Slepička, P.; Zbytovská, J.; Vávrová, K. Acidic pH Is Required for the Multilamellar Assembly of Skin Barrier Lipids In Vitro. J. Invest. Dermatol. 2021, 141, 1915-1921.e4.

(39) Lambers, H.; Piessens, S.; Bloem, A.; Pronk, H.; Finkel, P. Natural skin surface $\mathrm{pH}$ is on average below 5 , which is beneficial for its resident flora. Int. J. Cosmet. Sci. 2006, 28, 359-370.

(40) Maleki Dizaj, S.; Barzegar-Jalali, M.; Zarrintan, M. H.; Adibkia, K.; Lotfipour, F. Calcium carbonate nanoparticles as cancer drug delivery system. Expert Opin. Drug Deliv. 2015, 12, 1649-1660.

(41) Dash, S.; Murthy, P. N.; Nath, L.; Chowdhury, P. Kinetic modeling on drug release from controlled drug delivery systems. Acta Pol. Pharm. 2010, 67, 217-223.

(42) Desai, P.; Patlolla, R. R.; Singh, M. Interaction of nanoparticles and cell-penetrating peptides with skin for transdermal drug delivery. Mol. Membr. Biol. 2010, 27, 247-259.

(43) Patel, V.; Sharma, O. P.; Mehta, T. Nanocrystal: a novel approach to overcome skin barriers for improved topical drug delivery. Expert Opin. Drug Deliv. 2018, 15, 351-368. 